\title{
COMPATIBILITY STUDY BETWEEN PRAVASTATIN AND EZETIMIBE, AND PHARMACEUTICAL EXCIPIENTS USED IN FIXED-DOSE COMBINATION TABLET
}

\section{KANG MIN KIM*}

Department of Pharmaceutical Science and Technology, Kyungsung University, Busan, South Korea. Email: kimkmks@ks.ac.kr Received: 14 April 2021, Revised and Accepted: 11 June 2021

\begin{abstract}
Objective: The purpose of this study was to qualitatively predict drug-excipient binding interactions for stable drug formulation of a pravastatin and ezetimibe fixed-dose combination (FDC) tablet.

Methods: Drug impurity-excipient interactions under accelerated conditions $\left(40^{\circ} \mathrm{C} / 75 \%\right.$ relative humidity) for 4 weeks were confirmed by highperformance liquid chromatography, X-ray diffraction (XRD), differential scanning calorimetry (DSC) and thermogravimetric (TG) analysis.

Results: Pravastatin impurity was affected by four excipients under accelerated conditions for 4 weeks. Ezetimibe was affected by two excipients. Any other results were within the acceptance criteria. XRD analysis for physical stability revealed characteristic peaks of pravastatin and ezetimibe at a diffraction angle of $2 \theta$ (pravastatin: $4.1-24.4^{\circ}$, and ezetimibe: $13.62-29.59^{\circ}$ ) without a change in the crystalline form after 4 weeks. DSC and TG analysis showed evidence of stability in Alu-Alu foil.
\end{abstract}

Conclusion: Thus, the tested excipients were confirmed to be compatible with pravastatin and ezetimibe and can be used in FDC bi-layer tablets.

Keywords: Fixed-dose combination tablet, Compatibility, Pravastatin, Ezetimibe.

(C) 2021 The Authors. Published by Innovare Academic Sciences Pvt Ltd. This is an open access article under the CC BY license (http://creativecommons.org/ licenses/by/4.0/) DOI: http://dx.doi.org/10.22159/ajpcr.2021v14i8.41793. Journal homepage: https://innovareacademics.in/journals/index.php/ajpcr

\section{INTRODUCTION}

Pravastatin is a 3-hydroxy-3-methylglutaryl coenzyme A reductase inhibitor that lowers serum cholesterol levels and reduces cardiovascular risk [1]. Statin-based drugs are actively recommended when patients with increased cholesterol have high blood pressure, diabetes, and cardiovascular disease [2]. Following oral administration, pravastatin reaches maximum plasma concentration $\left(\mathrm{C}_{\max }\right)$ after approximately $0.88-1.00 \mathrm{~h}$ and has a half-life of $1.97-2.15 \mathrm{~h}$ and $18 \%$ bioavailability [3]. Ezetimibe inhibits Niemann-Pick C1-Like one protein affecting intestinal cholesterol absorption [4]. Ezetimibe reaches $\mathrm{C}_{\max }$ at approximately 1.00$2.00 \mathrm{~h}$ after oral administration; its half-life is approximately $22.00 \mathrm{~h}$ with low and high oral bioavailability of $35 \%$ and $65 \%$, respectively $[5,6]$.

Fixed-dose combination (FDC) tablets include various additives such as excipients, binders, disintegrants, and lubricants besides the active pharmaceutical ingredient (API) to ensure adequate absorption, improve appearance, and increase stability. According to the general rule of the Korean Pharmacopoeia, appropriate additives such as excipients, stabilizers, preservatives, and buffering agents may be added to the formulation, unless otherwise stipulated, to secure quality and enhance efficacy. All additives should be harmless to the API dose and should not modify its therapeutic effect. Therefore, when formulating pharmaceuticals, additives should be selected considering their stability, safety, and quality. However, there may be interactions between the API and additives, such as complex formation or acidbase reactions, which may affect stability, dissolution rate, solubility, and bioavailability [7]. Thus, the study of potential physical and chemical interactions between drugs and excipients is an important step in the formulation process [8]. The recent International Council for Harmonization of Technical Requirements for Pharmaceuticals for Human Use Q8 (R2) guidelines on pharmaceutical development encourages the manufacture of stable medicines using the quality by design ( $\mathrm{QbD}$ ) approach [9]. In Korea, $\mathrm{QbD}$ is applied to each formulation and is included in the common technical document. Results of the compatibility tests between the API and the excipient are also included.
Thus, the purpose of this study was to investigate the compatibility of the excipients used in the development of a pravastatin and ezetimibe FDC tablet using high-performance liquid chromatography (HPLC), X-ray diffraction (XRD), differential scanning calorimetry (DSC), and thermogravimetric (TG) analysis. The excipients tested include lactose monohydrate, microcrystalline cellulose, magnesium oxide, hydrated ferric oxide, croscarmellose sodium, magnesium stearate, sodium lauryl sulfate, and polyvinylpyrrolidone.

\section{MATERIALS AND METHODS}

\section{Materials}

Pravastatin ( $>99 \%$ purity) was supplied by Guangdong Blue Treasure Pharmaceutical Co., Ltd. (Guangdong, China). Ezetimibe (>99\% purity) was supplied by Neuland Laboratories Limited (Andhra Pradesh, India). The excipients lactose monohydrate (Pharmatose ${ }^{\circledR}$ 200M, DMV Fonterra), microcrystalline cellulose (Vivapur $^{\circledR}$ 101), magnesium oxide (heavy), hydrated ferric oxide, croscarmellose sodium $\left(\right.$ Acdisol $\left.^{\circledR}\right)$, magnesium stearate $\left(\mathrm{Hyqual}^{\circledR}\right)$, sodium lauryl sulfate, and polyvinylpyrrolidone (PVP K30) were purchased from Hwawon Pharmaceuticals and Masung Co., Ltd. All reagents were of analytical or HPLC grade and were purchased from Merck (Whitehouse Station, NJ, USA). The reference formulation of pravastatin was Mevalotin ${ }^{\circledR}$ (Daiichi Sankyo Co. Ltd, Japan) and of ezetimibe was Ezetrol ${ }^{\circledR}$ (MSD Korea Co., Ltd., Korea).

\section{Drug-excipient compatibility study}

To assess compatibility, pravastatin, ezetimibe, and pharmaceutical excipients were mixed at an arbitrary weight ratio to confirm the stability of the interaction $[10,11]$. The excipients were used in a similar to that in the Mevalotin ${ }^{\circledR}$ and Ezetrol ${ }^{\circledR}$. Table 1 shows the test conditions for the compatibility study. Table 2 describes the ratios of the binary mixtures and whole and partial blends that were prepared to identify the interactions between the drugs (pravastatin and ezetimibe) and excipients [12]. The expected pravastatin and ezetimibe FDC tablet was double-layered and formulated through wet granulation (PG) (Table 3). 
All mixtures were prepared to form dry powders and were packed using Alu-Alu foil.

\section{Impurity test}

The impurities of pravastatin and ezetimibe were assessed using the Agilent 1100 series HPLC system (Agilent Technologies, USA). HPLC analysis for pravastatin was performed using a C18 column (Zorbax SB-C18, $4.6 \times 150 \mathrm{~mm}, 3.5-\mu \mathrm{m}$ particle size, Agilent Technologies). The mobile phase was injected by gradient elution and consisted of solvent A (distilled water: phosphate buffer $(\mathrm{pH}$ adjusted to 7.0 with trimethylamine in $0.08 \mathrm{~mol} / \mathrm{L}$ phosphoric acid):acetonitrile at $520: 300: 180 \mathrm{v} / \mathrm{v} / \mathrm{v}$ ) and solvent B (acetonitrile: phosphate buffer $(0.08 \mathrm{~mol} / \mathrm{L}, \mathrm{pH} 7.0):$ distilled water at $600: 300: 100 \mathrm{v} / \mathrm{v} / \mathrm{v})$ at a flow rate of $1.0 \mathrm{~mL} / \mathrm{min}$. The initial mobile phase composition was maintained at $100 \%$ solvent A for $3.00 \mathrm{~min}$, changed linearly to $0 \%$ (3.00-26.50 min), and maintained for $3.40 \mathrm{~min}$ (26.60-30.00 $\mathrm{min}$ ) for column equilibrium. The analysis time and detection wavelength were $30 \mathrm{~min}$ and $238 \mathrm{~nm}$, respectively. The standard stock solution was prepared by dissolving $12.5 \mathrm{mg}$ of pravastatin 1,1,3,3-tetramethylbutylamine standard in $100 \mathrm{~mL}$ of diluted $50 \%$ methanol. Then, $1.0 \mathrm{~mL}$ of this solution was diluted in $100 \mathrm{~mL}$ of $50 \%$ methanol, which was used as the standard solution. To prepare sample solution, $50 \mathrm{mg}$ of pravastatin was placed in a $100-\mathrm{mL}$ volumetric flask, to which $50 \%$ methanol was added; the flask was then shaken for 15-20 min in an ultrasonic shaker. Oxidation impurities, 6'-epipravastatin, pravastatin lactone, and other individual impurities as well as total impurities were identified [13].

HPLC analysis for ezetimibe was performed using a C18 column (Pursuit XRs C18, $250 \times 4.6 \mathrm{~mm}, 5-\mu \mathrm{m}$ particle size, Agilent Technologies). The mobile phase included gradient elution of acetonitrile and buffer $(0.05 \% \mathrm{w} / \mathrm{v}$ 1-heptanesulfonic acid sodium salt in $1000 \mathrm{~mL}$ of distilled water, adjusted to $\mathrm{pH} 6.8$ with $0.04 \% \mathrm{Na}_{2} \mathrm{CO}_{3}$ solution). The mobile phase consisted of solvent A $(0.05 \%[\mathrm{w} / \mathrm{v}]$ 1-heptanesulfonic acid

Table 1: Summary for the compatibility study of pravastatin and excipients

\begin{tabular}{|c|c|}
\hline Test items & Conditions \\
\hline Stability- & Qualitative analysis by HPLC (impurity), DSC- \\
\hline indicating method & $\mathrm{TG}^{\mathrm{a}}$, and description (visual) \\
\hline Storage condition & Accelerated $\left(40^{\circ} \mathrm{C} / 75 \%\right.$ Relative humidity) \\
\hline $\begin{array}{l}\text { Closure system } \\
\text { (open or closed) }\end{array}$ & Alu-Alu (closed) \\
\hline Test period & 4 weeks (sampling time $=0,2$, and 4 weeks) \\
\hline Mixing method & Binary mixtures, whole and partial blends \\
\hline
\end{tabular}

sodium salt buffer [pH 6.8]) and solvent B (acetonitrile) injected at a flow rate of $1.5 \mathrm{~mL} / \mathrm{min}$. The initial mobile phase composition was maintained at $70 \%$ solvent A, changed linearly to $60 \%$ (0.00-6.00 min), $55 \%$ (6.00-16.01 $\mathrm{min})$, and 10\% (16.01-28.00 $\mathrm{min})$, then maintained at $10 \%(28.00-30.00 \mathrm{~min})$, again changed linearly to $70 \%(30.00-$ $30.01 \mathrm{~min}$ ), and maintained for $10 \mathrm{~min}$ (30.01-40.00 $\mathrm{min}$ ) for column equilibrium. The analysis time and detection wavelength were $45 \mathrm{~min}$ and $235 \mathrm{~nm}$, respectively. For the standard solution, 25-mg ezetimibe standard was transferred to a $50 \mathrm{~mL}$ volumetric flask, dissolved in $20 \mathrm{~mL}$ of diluent (solvent $\mathrm{A}$ : solvent $\mathrm{B}=4: 6$ ), made up to the required volume with diluent, and shaken for 15-20 min in an ultrasonic shaker. Impurity-A, Impurity-B, Impurity-C, Impurity-D, and other individual impurities were identified on chromatograms [14].

\section{XRD analysis}

Diffractograms were obtained using an automated multipurpose X-ray diffractometer (SmartLab, Rigaku, Japan) with $\mathrm{Cu}-\mathrm{K}_{2}{ }^{\alpha}$ radiation $(40 \mathrm{kV}$, $40 \mathrm{~mA}$ ) in the range of $5-40^{\circ}(2 \theta)[15]$.

\section{DSC and TG}

DSC and TG were performed using a heat flux plate-type calorimeter (DSC 131 EVO, Setaram Instrumentation, France) and a thermobalance (N-1500, SCINCO, South Korea), respectively, at a temperature of 25$350^{\circ} \mathrm{C}$. Samples of approximately $4 \mathrm{mg}$ were assessed under nitrogen gas at a flow rate of $30 \mathrm{cc} / \mathrm{min}$ and a heating rate of $10^{\circ} \mathrm{C} / \mathrm{min}$.

\section{Statistical analysis}

Mean \pm standard deviation for all experimental results was evaluated using the SAS Software (Ver. 9.4, SAS Institute Inc., Cary, NC, USA).

\section{RESULTS}

\section{Drug-excipient compatibility}

The impurity content of pravastatin and ezetimibe changed when mixed with excipients. The accelerated test results of pravastatin indicated that all excipients except the PL, PC, PP, and PS samples (Table 2) of lactose monohydrate, croscarmellose sodium, polyvinylpyrrolidone, and sodium lauryl sulfate, respectively, were found to slightly increase total impurity (Table 4). The accelerated test results of ezetimibe indicated that except for the EM2, EP, and EO samples (Table 2) of magnesium oxide, polyvinylpyrrolidone, and ezetimibe only, other excipients did not increase total impurity (Table 5). However, all observed impurity results were below the acceptance criteria, as shown in Tables 4 and 5 .

\section{Powder XRD analysis}

The XRD curves of drugs and excipients (Table 2) revealed characteristic peaks showing crystalline forms. The XRD patterns of pravastatin and ezetimibe (Fig. 1) showed sharp, intense, and less diffused peaks at $2 \theta$ angles (pravastatin: $4.1-24.4^{\circ}$ and ezetimibe: $13.62-29.59^{\circ}$ ), indicating

Table 2: Samples for drug-excipient compatibility study

\begin{tabular}{|c|c|c|c|c|c|c|}
\hline \multirow{3}{*}{$\begin{array}{l}\text { Drug substance (A) } \\
\text { Pravastatin sodium } \\
\text { (P) and Ezetimibe (E) }\end{array}$} & \multirow{2}{*}{$\begin{array}{l}\text { Excipients (B) } \\
\text { Lactose monohydrate }\end{array}$} & \multirow{2}{*}{$\begin{array}{l}\text { Samples } \\
\text { PL, EL }\end{array}$} & \multicolumn{2}{|c|}{ A:B ratio (Pravastatin) } & \multicolumn{2}{|c|}{ A:B ratio (Ezetimibe) } \\
\hline & & & 1 & 10 & 1 & 14 \\
\hline & Microcrystalline cellulose & PM1, EM1 & 1 & 4 & 1 & 4 \\
\hline & Magnesium oxide & PM2, EM2 & 2 & 1 & 2 & 1 \\
\hline & Hydrated ferric oxide & $\mathrm{PH}, \mathrm{EH}$ & 200 & 1 & 50 & 1 \\
\hline & Croscarmellose sodium & $\mathrm{PC}, \mathrm{EC}$ & 1 & 4 & 1 & 6 \\
\hline & Polyvinylpyrrolidone & PP, EP & 5 & 1 & 5 & 1 \\
\hline & Sodium lauryl sulfate & PS, ES & 2 & 1 & 2 & 1 \\
\hline & Magnesium stearate & PM3, EM3 & 4 & 1 & 4 & 1 \\
\hline \multicolumn{2}{|c|}{ Pravastatin granule after wet $\mathrm{PG}^{\mathrm{a}}$} & PG & $\mathrm{N} / \mathrm{A}$ & & & \\
\hline \multicolumn{2}{|c|}{ Ezetimibe granule after wet $\mathrm{PG}^{\mathrm{b}}$} & EG & N/A & & & \\
\hline \multicolumn{2}{|c|}{ Pravastatin total mixture ${ }^{c}$} & PTM & $\mathrm{N} / \mathrm{A}$ & & & \\
\hline \multicolumn{2}{|l|}{ Ezetimibe total mixture $^{\mathrm{d}}$} & ETM & $\mathrm{N} / \mathrm{A}$ & & & \\
\hline \multicolumn{2}{|l|}{ Pravastatin only } & PO & $\mathrm{N} / \mathrm{A}$ & & & \\
\hline \multicolumn{2}{|l|}{ Ezetimibe only } & EO & N/A & & & \\
\hline
\end{tabular}

aPravastatin wet granulation is mixed and performed by ingredients of No. 1-5. 'bzetimibe wet granulation is mixed and performed by ingredients of No. $1,2,5,6$, and 7. ${ }^{c}$ Pravastatin total mixture is performed by ingredients of No. 1-5, and 8. dEzetimibe total mixture is performed by ingredients of No. 1, 2, 5, and 6-8. The composition of pravastatin and ezetimibe wet granulation and total mixture is as Table 3, PG: Granulation 
the high crystalline form of pure pravastatin (PO sample) (Fig. 1a) and ezetimibe (EO sample) (Fig. 1b). After maintaining accelerated condition $\left(40^{\circ} \mathrm{C}\right.$. Af relative humidity) for 4 weeks (Fig. 1c and d), the diffraction patterns of the drugs and excipients showed several peaks similar to the original form, indicating that the crystallinity of pravastatin and ezetimibe remained unchanged (Fig. 1).

\section{DSC and TG}

The results of DSC and TG of pravastatin (PO sample of Table 2) and ezetimibe (EO sample of Table 2) in Alu-Alu foil under accelerated conditions are illustrated in Fig. 2. The initial DSC curve of pravastatin showed the first endothermic event between 174 and $188^{\circ} \mathrm{C}\left(\Delta \mathrm{H}_{\text {fusion }}\right.$ $1.394 \mathrm{~J}$ ), with a melting temperature of $\mathrm{T}_{\text {onset }}$ of $174^{\circ} \mathrm{C}$ (Fig. 2a). The TG

Table 3: The expected formulation of pravastatin and ezetimibe layers

\begin{tabular}{lll}
\hline Ingredient & $\begin{array}{l}\text { Pravastatin } \\
\text { formulation (mg) }\end{array}$ & $\begin{array}{l}\text { Ezetimibe } \\
\text { formulation (mg) }\end{array}$ \\
\hline Pravastatin & 40.00 & - \\
Ezetimibe & - & 10.00 \\
Lactose monohydrate & 91.00 & 43.50 \\
Microcrystalline cellulose & 19.80 & 20.00 \\
Magnesium oxide & 7.00 & - \\
Hydrated ferric oxide & 0.20 & - \\
Polyvinylpyrrolidone & 10.00 & 2.00 \\
Sodium lauryl sulfate & - & 4.00 \\
Croscarmellose sodium & 30.00 & 20.00 \\
Magnesium stearate & 2.00 & 0.50 \\
Total & 200 & 100 \\
\hline
\end{tabular}

curve exhibited $42.574 \%$ mass loss between 249 and $385^{\circ} \mathrm{C}$ with the decomposition of pravastatin (Fig. 2c). The DSC curve of pravastatin after 4 weeks showed the first endothermic event between 166 and $179^{\circ} \mathrm{C}\left(\Delta \mathrm{H}_{\text {fusion }}: 1.079 \mathrm{~J}\right)$, with a melting temperature of $\mathrm{T}_{\text {onset }}$ of $166^{\circ} \mathrm{C}$ (Fig. 2a). The TG curve exhibited $41.130 \%$ mass loss between 251 and $388^{\circ} \mathrm{C}$ with the decomposition of pravastatin (Fig. 2c).

The initial DSC curve of ezetimibe showed the first endothermic event between 162 and $173^{\circ} \mathrm{C}\left(\Delta \mathrm{H}_{\text {fusion }}: 2.769 \mathrm{~J}\right)$, with a melting temperature of $\mathrm{T}_{\text {onset }}$ of $162^{\circ} \mathrm{C}$ (Fig. 2b). The TG curve exhibited $90.38 \%$ mass loss between 190 and $392^{\circ} \mathrm{C}$ (Fig. 2d). The DSC curve of ezetimibe after 4 weeks showed the first endothermic event between 161 and $170^{\circ} \mathrm{C}$ $\left(\Delta \mathrm{H}_{\text {fusion }}: 1.079 \mathrm{~J}\right)$, with a melting temperature of $\mathrm{T}_{\text {onset }}$ of $161^{\circ} \mathrm{C}$ (Fig. $\left.2 \mathrm{~b}\right)$. The TG curve exhibited $89.19 \%$ mass loss between 202 and $394^{\circ} \mathrm{C}$ (Fig. 2d).

\section{DISCUSSION}

This study tested the compatibility of excipients used in a bi-layer FDC tablet of pravastatin and ezetimibe. The total weight of Ezetrol ${ }^{\circledR}$ (ezetimibe) $10 \mathrm{mg}$ tablet was $100 \mathrm{mg}$ and of Mevalotin ${ }^{\circledR}$ (pravastatin) $40 \mathrm{mg}$ was $400 \mathrm{mg}$. The total weight of the ezetimibe layer for the FDC tablet retained the same amount as Ezetrol ${ }^{\circledR} 10 \mathrm{mg}$. However, $200 \mathrm{mg}$ of the total weight of the pravastatin layer was used to improve dose compliance. An understanding of compatibility is an integral part of the pre-formulation stage to assess safety, therapeutic properties, and stability of the dosage form. Hence, compatibility of pravastatin and ezetimibe of the bi-layer tablet should be studied with the excipients used in the other layer [16]. General excipients were used in this study to identify their stability.

Table 4: Compatibility results on each binary and all mixture of pravastatin with excipients under $40^{\circ} \mathrm{C} / 75 \%$ relative humidity condition

\begin{tabular}{|c|c|c|c|c|c|c|c|}
\hline Samples & $\begin{array}{l}\text { Time } \\
\text { (Weeks) }\end{array}$ & $\begin{array}{l}\text { Oxidation } \\
\text { impurity }(<1 \%)\end{array}$ & $\begin{array}{l}\text { Impurity B } \\
(<0.3 \%)\end{array}$ & $\begin{array}{l}\text { Pravastatin } \\
\text { lactone }(<2 \%)\end{array}$ & $\begin{array}{l}\text { Any other individual } \\
\text { impurity }(<0.2 \%)\end{array}$ & $\begin{array}{l}\text { Total impurity } \\
(<0.5 \%)\end{array}$ & Description \\
\hline \multirow[t]{3}{*}{$\mathrm{PL}$} & 0 & $<\mathrm{RT}^{\mathrm{a}}$ & $<\mathrm{RT}$ & $<\mathrm{RT}$ & $<\mathrm{RT}$ & $<\mathrm{RT}$ & White or off white powder \\
\hline & 2 & $<\mathrm{RT}$ & $<\mathrm{RT}$ & $<\mathrm{RT}$ & $<\mathrm{RT}$ & $<\mathrm{RT}$ & White or off white powder \\
\hline & 4 & $<\mathrm{RT}$ & $<\mathrm{RT}$ & $<\mathrm{RT}$ & $<\mathrm{RT}$ & $<\mathrm{RT}$ & White or off white powder \\
\hline \multirow[t]{3}{*}{ PM1 } & 0 & $<\mathrm{RT}$ & $<\mathrm{RT}$ & $<\mathrm{RT}$ & $<\mathrm{RT}$ & $<\mathrm{RT}$ & White or off white powder \\
\hline & 2 & $<\mathrm{RT}$ & $<\mathrm{RT}$ & $<\mathrm{RT}$ & $<\mathrm{RT}$ & $<\mathrm{RT}$ & White or off white powder \\
\hline & 4 & $<\mathrm{RT}$ & $<\mathrm{RT}$ & $<\mathrm{RT}$ & $<\mathrm{RT}$ & $<\mathrm{RT}$ & White or off white powder \\
\hline \multirow[t]{3}{*}{ PM2 } & 0 & $<\mathrm{RT}$ & $<\mathrm{RT}$ & $<\mathrm{RT}$ & $<\mathrm{RT}$ & $<$ RT $0.11 \pm 0.007$ & White or off white powder \\
\hline & 2 & $<\mathrm{RT}$ & $<\mathrm{RT}$ & $<\mathrm{RT}$ & $0.11 \pm 0.007$ & $0.12 \pm 0.004$ & White or off white powder \\
\hline & 4 & $<\mathrm{RT}$ & $<\mathrm{RT}$ & $<\mathrm{RT}$ & $0.12 \pm 0.004$ & & White or off white powder \\
\hline \multirow[t]{3}{*}{$\mathrm{PH}$} & 0 & $<\mathrm{RT}$ & $<\mathrm{RT}$ & $<\mathrm{RT}$ & $<\mathrm{RT}$ & $<$ RT $0.10 \pm 0.003$ & Light brown powder \\
\hline & 2 & $<\mathrm{RT}$ & $<\mathrm{RT}$ & $<\mathrm{RT}$ & $0.10 \pm 0.003$ & $0.12 \pm 0.006$ & Light brown powder \\
\hline & 4 & $<\mathrm{RT}$ & $<\mathrm{RT}$ & $<\mathrm{RT}$ & $0.12 \pm 0.006$ & & Light brown powder \\
\hline \multirow[t]{2}{*}{ PC } & 0 & $<\mathrm{RT}$ & $<\mathrm{RT}$ & $<\mathrm{RT}$ & $<\mathrm{RT}$ & $<\mathrm{RT}$ & White or off white powder \\
\hline & 2 & $<\mathrm{RT}$ & $<\mathrm{RT}$ & $<\mathrm{RT}$ & $<\mathrm{RT}$ & $<\mathrm{RT}$ & White or off white powder \\
\hline \multirow[t]{3}{*}{ PP } & 0 & $<\mathrm{RT}$ & $<\mathrm{RT}$ & $<\mathrm{RT}$ & $<\mathrm{RT}$ & $<\mathrm{RT}$ & White or off white powder \\
\hline & 2 & $<\mathrm{RT}$ & $<\mathrm{RT}$ & $<\mathrm{RT}$ & $<\mathrm{RT}$ & $<\mathrm{RT}$ & White or off white powder \\
\hline & 4 & $<\mathrm{RT}$ & $<\mathrm{RT}$ & $<\mathrm{RT}$ & $<\mathrm{RT}$ & $<\mathrm{RT}$ & White or off white powder \\
\hline \multirow[t]{3}{*}{ PS } & 0 & $<\mathrm{RT}$ & $<\mathrm{RT}$ & $<\mathrm{RT}$ & $<\mathrm{RT}$ & $<\mathrm{RT}$ & White or off white powder \\
\hline & 2 & $<\mathrm{RT}$ & $<\mathrm{RT}$ & $<\mathrm{RT}$ & $<\mathrm{RT}$ & $<\mathrm{RT}$ & White or off white powder \\
\hline & 4 & $<\mathrm{RT}$ & $<\mathrm{RT}$ & $<\mathrm{RT}$ & $<\mathrm{RT}$ & $<\mathrm{RT}$ & White or off white powder \\
\hline \multirow[t]{3}{*}{ PM3 } & 0 & $<\mathrm{RT}$ & $<\mathrm{RT}$ & $<\mathrm{RT}$ & $<\mathrm{RT}$ & $<\mathrm{RT}$ & White or off white powder \\
\hline & 2 & $<\mathrm{RT}$ & $<\mathrm{RT}$ & $<\mathrm{RT}$ & $<\mathrm{RT}$ & $<\mathrm{RT}$ & White or off white powder \\
\hline & 4 & $<\mathrm{RT}$ & $<\mathrm{RT}$ & $<\mathrm{RT}$ & $<\mathrm{RT}$ & $<\mathrm{RT}$ & White or off white powder \\
\hline \multirow[t]{3}{*}{$P G$} & 0 & $<\mathrm{RT}$ & $<\mathrm{RT}$ & $<\mathrm{RT}$ & $0.11 \pm 0.008$ & $0.11 \pm 0.008$ & White or off white powder \\
\hline & 2 & $<\mathrm{RT}$ & $<\mathrm{RT}$ & $<\mathrm{RT}$ & $0.12 \pm 0.007$ & $0.12 \pm 0.007$ & White or off white powder \\
\hline & 4 & $<\mathrm{RT}$ & $<\mathrm{RT}$ & $<\mathrm{RT}$ & $0.13 \pm 0.008$ & $0.13 \pm 0.006$ & White or off white powder \\
\hline \multirow[t]{3}{*}{ PTM } & 0 & $<\mathrm{RT}$ & $<\mathrm{RT}$ & $<\mathrm{RT}$ & $<\mathrm{RT}$ & $<\mathrm{RT}$ & White or off white powder \\
\hline & 2 & $<\mathrm{RT}$ & $<\mathrm{RT}$ & $<\mathrm{RT}$ & $0.10 \pm 0.003$ & $<\mathrm{RT}$ & White or off white powder \\
\hline & 4 & $<\mathrm{RT}$ & $<\mathrm{RT}$ & $<\mathrm{RT}$ & $0.11 \pm 0.006$ & $0.11 \pm 0.006$ & White or off white powder \\
\hline PO & 0 & $<\mathrm{RT}$ & $<\mathrm{RT}$ & $<\mathrm{RT}$ & $<\mathrm{RT}$ & $<\mathrm{RT}$ & White or off white powder \\
\hline
\end{tabular}

aReporting thresholds $(0.1 \%)$ 
Table 5: Compatibility results on each binary and all mixture of ezetimibe with excipients under $40^{\circ} \mathrm{C} / 75 \%$ relative humidity condition

\begin{tabular}{|c|c|c|c|c|c|c|}
\hline Samples & $\begin{array}{l}\text { Time } \\
\text { (Weeks) }\end{array}$ & $\begin{array}{l}\text { Impurity B } \\
(<0.3 \%)\end{array}$ & $\begin{array}{l}\text { Impurity E } \\
(<0.2 \%)\end{array}$ & $\begin{array}{l}\text { Any other individual } \\
\text { impurity }(<0.2 \%)\end{array}$ & $\begin{array}{l}\text { Total impurity } \\
(<0.5 \%)\end{array}$ & Description \\
\hline \multirow[t]{3}{*}{ EL } & 0 & $0.01 \pm 0.005$ & $<\mathrm{RT}$ & $<\mathrm{RT}$ & $0.01 \pm 0.001$ & White or off white powder \\
\hline & 2 & $0.02 \pm 0.005$ & $0.03 \pm 0.008$ & $<\mathrm{RT}$ & $0.06 \pm 0.001$ & White or off white powder \\
\hline & 4 & $0.01 \pm 0.008$ & $0.03 \pm 0.008$ & $<\mathrm{RT}$ & $0.05 \pm 0.008$ & White or off white powder \\
\hline \multirow[t]{3}{*}{ EM1 } & 0 & $0.04 \pm 0.006$ & $0.02 \pm 0.002$ & $<\mathrm{RT}$ & $0.08 \pm 0.008$ & White or off white powder \\
\hline & 2 & $0.04 \pm 0.009$ & $0.02 \pm 0.004$ & $<\mathrm{RT}$ & $0.07 \pm 0.003$ & White or off white powder \\
\hline & 4 & $0.04 \pm 0.007$ & $0.02 \pm 0.003$ & $<\mathrm{RT}$ & $0.07 \pm 0.004$ & White or off white powder \\
\hline \multirow[t]{3}{*}{ EM2 } & 0 & $0.06 \pm 0.007$ & $0.02 \pm 0.009$ & $0.01 \pm 0.005$ & $0.11 \pm 0.002$ & White or off white powder \\
\hline & 2 & $0.07 \pm 0.007$ & $0.03 \pm 0.007$ & $0.02 \pm 0.007$ & $0.12 \pm 0.003$ & White or off white powder \\
\hline & 4 & $0.08 \pm 0.009$ & $0.06 \pm 0.007$ & $0.04 \pm 0.007$ & $0.18 \pm 0.009$ & White or off white powder \\
\hline \multirow[t]{3}{*}{ EH } & 0 & $0.03 \pm 0.006$ & $0.02 \pm 0.004$ & $<\mathrm{RT}$ & $0.05 \pm 0.001$ & Light brown powder \\
\hline & 2 & $0.03 \pm 0.004$ & $0.02 \pm 0.003$ & $<\mathrm{RT}$ & $0.05 \pm 0.007$ & Light brown powder \\
\hline & 4 & $0.03 \pm 0.008$ & $0.01 \pm 0.008$ & $<\mathrm{RT}$ & $0.05 \pm 0.006$ & Light brown powder \\
\hline \multirow[t]{3}{*}{ EC } & 0 & $<\mathrm{RT}$ & $<\mathrm{RT}$ & $<\mathrm{RT}$ & $<\mathrm{RT}$ & White or off white powder \\
\hline & 2 & $<\mathrm{RT}$ & $<\mathrm{RT}$ & $<\mathrm{RT}$ & $<\mathrm{RT}$ & White or off white powder \\
\hline & 4 & $<\mathrm{RT}$ & $<\mathrm{RT}$ & $<\mathrm{RT}$ & $<\mathrm{RT}$ & White or off white powder \\
\hline \multirow[t]{3}{*}{ EP } & 0 & $0.02 \pm 0.001$ & $<\mathrm{RT}$ & $0.06 \pm 0.008$ & $0.08 \pm 0.009$ & White or off white powder \\
\hline & 2 & $0.02 \pm 0.008$ & $0.01 \pm 0.009$ & $0.05 \pm 0.007$ & $0.10 \pm 0.003$ & White or off white powder \\
\hline & 4 & $0.02 \pm 0.002$ & $0.01 \pm 0.008$ & $0.06 \pm 0.007$ & $0.11 \pm 0.009$ & White or off white powder \\
\hline \multirow[t]{3}{*}{ ES } & 0 & $0.02 \pm 0.004$ & $<\mathrm{RT}$ & $<\mathrm{RT}$ & $0.03 \pm 0.001$ & White or off white powder \\
\hline & 2 & $0.02 \pm 0.007$ & $0.03 \pm 0.006$ & $<\mathrm{RT}$ & $0.06 \pm .0003$ & White or off white powder \\
\hline & 4 & $0.01 \pm 0.006$ & $0.04 \pm 0.004$ & $<\mathrm{RT}$ & $0.06 \pm 0.009$ & White or off white powder \\
\hline \multirow[t]{3}{*}{ EM3 } & 0 & $0.03 \pm 0.001$ & $0.02 \pm 0.005$ & $0.01 \pm 0.009$ & $0.06 \pm 0.005$ & White or off white powder \\
\hline & 2 & $0.03 \pm 0.005$ & $0.02 \pm 0.006$ & $0.01 \pm 0.001$ & $0.06 \pm 0.006$ & White or off white powder \\
\hline & 4 & $0.03 \pm 0.003$ & $0.02 \pm 0.006$ & $0.01 \pm 0.001$ & $0.06 \pm 0.009$ & White or off white powder \\
\hline \multirow[t]{3}{*}{ EG } & 0 & $0.02 \pm 0.002$ & $0.02 \pm 0.003$ & $<\mathrm{RT}$ & $0.05 \pm 0.006$ & White or off white powder \\
\hline & 2 & $0.02 \pm 0.009$ & $0.02 \pm 0.007$ & $<\mathrm{RT}$ & $0.05 \pm 0.006$ & White or off white powder \\
\hline & 4 & $0.02 \pm 0.008$ & $0.02 \pm 0.009$ & $<\mathrm{RT}$ & $0.05 \pm 0.009$ & White or off white powder \\
\hline \multirow[t]{3}{*}{ ETM } & 0 & $0.05 \pm 0.009$ & $0.02 \pm 0.004$ & $<\mathrm{RT}$ & $0.08 \pm 0.001$ & White or off white powder \\
\hline & 2 & $0.02 \pm 0.007$ & $0.03 \pm 0.005$ & $<\mathrm{RT}$ & $0.07 \pm 0.007$ & White or off white powder \\
\hline & 4 & $0.02 \pm 0.003$ & $0.03 \pm 0.008$ & $<\mathrm{RT}$ & $0.08 \pm 0.001$ & White or off white powder \\
\hline \multirow[t]{3}{*}{ EO } & 0 & $<\mathrm{RT}$ & $0.02 \pm 0.007$ & $<\mathrm{RT}$ & $0.03 \pm 0.004$ & White or off white powder \\
\hline & 2 & $0.03 \pm 0.003$ & $0.02 \pm 0.008$ & $<\mathrm{RT}$ & $0.06 \pm 0.005$ & White or off white powder \\
\hline & 4 & $0.02 \pm 0.006$ & $0.02 \pm 0.005$ & $<\mathrm{RT}$ & $0.06 \pm 0.001$ & White or off white powder \\
\hline
\end{tabular}

Some incompatible combinations of general excipients with known functional groups and their reactions are as follows: primary amine and mono- and disaccharides (amine-aldehyde or amine-acetal reactions); ester, cyclic, lactose, and basic compounds (ring-opening, ester-base or hydrolysis reactions); carbonyl, hydroxyl, and silanol (hydrogen bonding reactions); aldehyde, amine, and carbohydrates (aldehyde-amine, Schiff base or glycosylamine formation reactions); carboxyl and bases (salt formation reactions); alcohol and oxygen (oxidation of aldehydes and ketones); sulfhydryl and oxygen (dimerization reactions); phenol and metals (complexation reactions); and gelatin capsule and cationic surfactants (denaturation reactions). Therefore, incompatible combinations are generally excluded during formulation development [16]. As shown in Tables 4 and 5, pravastatin and ezetimibe impurities changed when mixed with excipients. Magnesium oxide and hydrated ferric oxide in the PM2 $(0.12 \pm 0.004 \%)$ and $\mathrm{PH}(0.12 \pm 0.004 \%)$ samples affected pravastatin impurities (total impurity) after 4 weeks (Table 4 ). These results affected the total impurity of wet PG and total mixture (PTM). However, all results were below the acceptance values. Morphologically, the drugs were not observed as lumps and did not exhibit browning (Table 4). Ezetimibe results showed low-level interactions except with magnesium oxide and polyvinylpyrrolidone in the EM2 (0.18 $0.009 \%)$ and EP $(0.11 \pm 0.009 \%)$ samples that affected ezetimibe impurities along with values of total impurity after 4 weeks (Table 5). Ezetimibe impurity and description were also within acceptance ranges. The binary mixtures PG, EG, PTM, and ETM had total impurity values of $0.13 \pm 0.006,0.05 \pm 0.009,0.11 \pm 0.006$, and $0.08 \pm 0.001 \%$, respectively, after 4 weeks under $40^{\circ} \mathrm{C}$ relative humidity conditions (Tables 4 and 5). Although, some excipients can have minor effects on pravastatin and ezetimibe stability, the formulation (wet PG process) indicated in Table 3 can be considered for the development of pravastatin and ezetimibe FDC tablets.

DSC or thin layer chromatography (TLC) is commonly used to select excipients. Moreover, excipients are selected for formulation studies if they show no interactions with the drug according to DSC results $[17,18]$. However, the DSC method is not sensitive to small changes, cannot confirm the effects of various environmental factors, and confirming long-term stability is difficult. Thus, decomposition products that cannot be identified using DSC are identified using TLC [17]. In this study, chemical stability between the drugs pravastatin and ezetimibe and excipients was evaluated by HPLC under accelerated conditions $\left(40^{\circ} \mathrm{C}\right.$ this study, chemicals for 4 weeks. XRD, DSC, and TG were used to assess the physical stability of pravastatin and ezetimibe [19].

The crystal form determined using XRD analysis was directly assessed to identify the stability of the drugs (pravastatin and ezetimibe) with

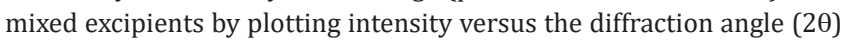
(Fig. 1). The results confirmed a single crystal form of pravastatin (PO sample) and ezetimibe (EO sample) [6,20]. The XRD analysis of drugs mixed with excipients demonstrated some characteristic peaks without unaltered drug peaks, indicating that a homogeneous mixture was achieved under accelerated conditions $\left(40^{\circ} \mathrm{C} / 75 \%\right.$ relative humidity) at 4 weeks (Fig. 1). In polymorphism studies of FDC tablet development, $\mathrm{XRD}$ analysis is important to select excipients compatible with the drug during processes such as direct compression, dry PG, and wet PG [21]. XRD studies also provide more information on DSC and TG results.

DSC and TG are commonly used to select excipients during pharmaceutical formulation of solid dosage forms [22]. DSC is used for 


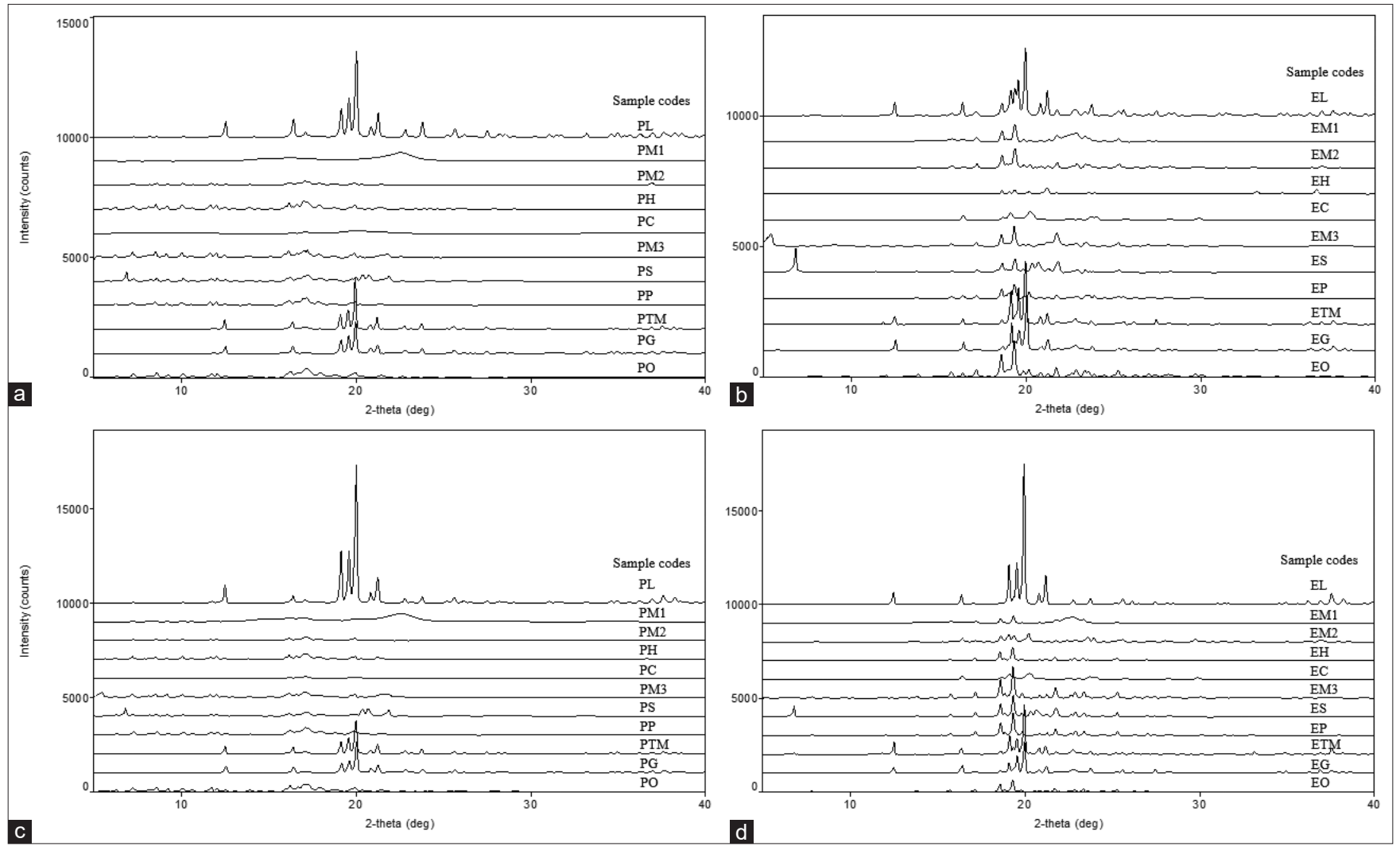

Fig. 1: Powder X-ray diffraction results on (a) pravastatin (initial), (b) ezetimibe (initial), (c) pravastatin (after 4 weeks), and (d) ezetimibe (after 4 weeks) under $40^{\circ} \mathrm{C} / 75 \%$ relative humidity condition. The name of samples is same as Table 2

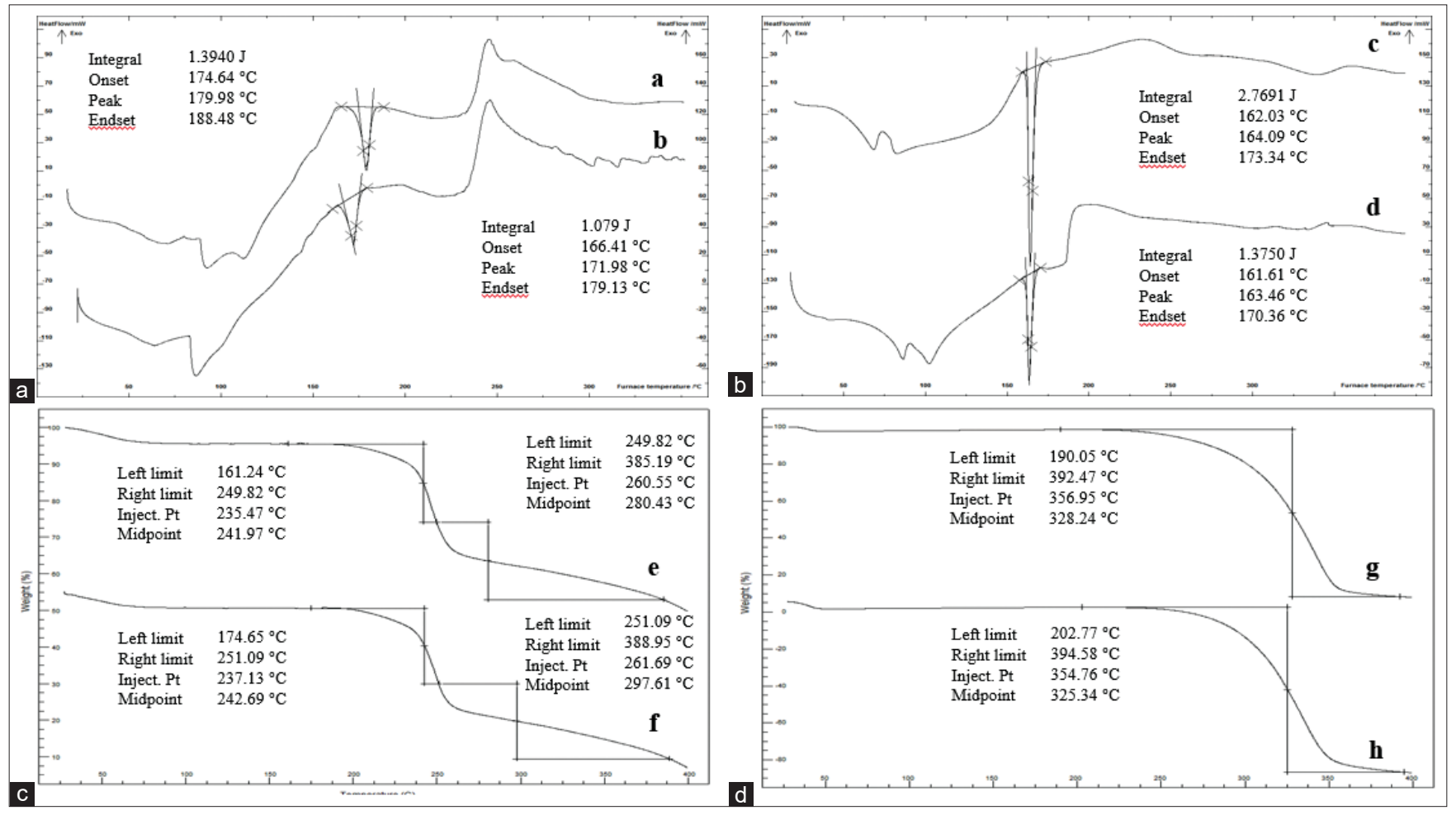

Fig. 2: Differential scanning calorimetry (DSC) and thermogravimetry (TG) curves of pravastatin and ezetimibe stored at accelerated $\left(40^{\circ} \mathrm{C} / 75 \%\right.$ Relative humidity) for initial (a) and (c) DSC (a for pravastatin and $\mathrm{c}$ for ezetimibe) and TG (e for pravastatin and $\mathrm{g}$ for ezetimibe), and after 4 weeks (b) and (d) DSC (b for pravastatin and d for ezetimibe) and TG (f for pravastatin and h for ezetimibe) 
analyzing and comparing physico-chemical interactions between drugs and excipients [23]. To determine the chemical stability of pravastatin and ezetimibe, their thermoanalytical profiles were identified by DSC and TG under accelerated conditions $\left(40^{\circ} \mathrm{C} / 75 \%\right.$ relative humidity) using initial and 4-weeks samples in Alu-Alu foil (PO and EO samples; Table 2). DSC and TG of pravastatin and ezetimibe in Alu-Alu foil for 4 weeks demonstrated no change in their thermoanalytical patterns (Fig. 2).

\section{CONCLUSION}

In this study, excipients compatible with pravastatin and ezetimibe were identified by impurity testing, XRD, DSC, and TG. We found that lactose monohydrate; croscarmellose sodium, polyvinylpyrrolidone, sodium lauryl sulfate, and magnesium oxide were slightly affected by the impurities of pravastatin and ezetimibe and were within acceptance ranges of total impurity. According to the results of XRD, DSC, and TG, the applicability of pravastatin and ezetimibe FDC tablets was demonstrated by a preformulation study. All excipients used in this study were compatible with pravastatin and ezetimibe. This study concluded that the tested excipients can be used for formulating pravastatin and ezetimibe FDC tablets.

\section{AUTHOR'S CONTRIBUTIONS}

Prof. K. M. Kim performed experiments, interpreted data, wrote the manuscript, and acted as corresponding author.

\section{CONFLICTS OF INTERESTS}

Authors have none to declare.

\section{FUNDING}

This research was supported by a Kyungsung University Research Grants in 2021

\section{REFERENCES}

1. Kivistö KT, Grisk O, Hofmann U, Meissner K, Möritz KU, Ritter C, et al. Disposition of oral and intravenous pravastatin in MRP2-deficient TRrats. Drug Metab Dispos 2005;33:1593-6.

2. Jeong YJ, Kim JM, Jang SJ, Bang JH, Jung YG, Kim ST, et al. The effect of pravastatin on insulin resistance in hyperglycemic patients. J Korean Diabetes 2017;18:53-61.

3. Bharate SS, Bharate SB, Bajaj AN. Incompatibilities of pharmaceutical excipients with active pharmaceutical ingredients: A comprehensive review. J Excipients Food Chem 2010;1:3-26.

4. Kosoglou T, Statkevich P, Johnson-Levonas AO, Paolini JF, Bergman AJ, Alton KB. Ezetimibe: A review of its metabolism, pharmacokinetics and drug interactions. Clin Pharmacokinet 2005;44:467-94.

5. Park JS, Kim CO, Jin BH, Yang SW, Park MS, Hong TG. Pharmacokinetic drug interaction between atorvastatin and ezetimibe in healthy Korean volunteers. Transl Clin Pharmacol 2017;25:202-8.

6. Torrado-Salmerón C, Guarnizo-Herrero V, Cerezo-Garreta J, Durán GT, Torrado-Santiago S. Self-micellizing technology improves the properties of ezetimibe and increases its effect on hyperlipidemic rats. Pharmaceutics 2019;11:647.

7. Kim KM. Compatibility study of excipients for pravastatin tablet. J Life Sci 2018;28:472-7

8. Kim KM. Formulation study for excipient compositions of pravastatin tablet. Lat Am J Pharm 2018;37:1709-17.

9. International Conference on Harmonization (ICH) Guideline Q8 (R2) Pharmaceutical Development; 2009.

10. Serajuddin AT, Thakur AB, Ghoshal RN, Fakes MG, Ranadive SA, Morris KR, et al. Selection of solid dosage form composition through drug-excipient compatibility testing. J Pharm Sci 1999;88:696-704.

11. Gao J, Fu X, Ding M, Fu Q. Studies on partial compatibility of PP and PS. Chin J Polym Sci 2010;28:647-56.

12. Ministry of Food and Drug Safety; 2015. Available from: http://www. mfds.go.kr/index.do?cd $=$ and searchkey $=$ title: contents and $\mathrm{mid}=914$ and pageno $=6$ and $\mathrm{seq}=22056$ and $\mathrm{cmd}=\mathrm{v}$.

13. United States Pharmacopeia. USP-41, NF-36, the Official Compendia of Standards, Pravastatin Sodium Tablets. United States: United States Pharmacopeia; 2018.

14. United States Pharmacopeia. USP-41, NF-36, the Official Compendia of Standards, Ezetimibe Tablets. United States: United States Pharmacopeia; 2018

15. Bertin EP. Principles and Practice of X-Ray Spectrometric Analysis. Netherlands: Kluwer Academic, Plenum Publishers; 2006. p. 321.

16. Malathi $\mathrm{P}$, Khan $\mathrm{AB}$. Recent approaches in bilayered technology: A review. Int J Pharm Sci 2012;3:4681-8.

17. Drug-Excipient Compatibility Studies; 2014. Available from: http:// www.pharmaquest.weebly.com/uploads/9/9/4/2/9942916/drug excipient_compatibility_study.pdf.

18. Sohn YT, Lee AK. Compatibility study using differential scanning calorimetry. J Korean Pharm Sci 1999;29:117-26.

19. Rus LM, Tomuta I, Iuga C, Maier C, Kacso I, Borodi G, et al. Compatibility studies of indapamide/pharmaceutical excipients used in tablet preformulation. Farmacia 2012;60:92-101.

20. Al-Badr AA, Mostafa GA. Pravastatin sodium. Profiles Drug Subst Excip Relat Methodol 2014;39:433-513.

21. Patel P, Ahir K, Patel V, Manani L, Patel C. Drug-excipient compatibility studies: First step for dosage form development. Pharma Innov 2015;4:14-20.

22. Stulzer HK, Rodrigues PO, Cardoso TM, Matos JS, Silva MA. Compatibility studies between captopril and pharmaceutical excipients used in tablets formulations. J Therm Anal Calorim 2008;91:323-8.

23. Rus L, Constantinescu D, Dragan F, Farcas A, Kacsó I, Borodi GH, et al. Inclusion complex of enalapril maleate/ $\beta$-cyclodextrin, FTIR, X-ray diffraction, DSC and molecular modeling. Farmacia 2007;55:185-92. 